\title{
Electron-Beam Micropatterning of Bioactive Surfaces
}

\author{
P. Krsko, M. Libera, R. Clancy*, and J. Kohn** \\ Stevens Institute of Technology, Hoboken, NJ \\ * Hospital for Joint Diseases, New York University, NY, NY \\ **Rutgers University, Piscataway, NJ
}

The fact that cell expression and differentiation is affected by the nature of its adhesion to a synthetic substrate has been demonstrated using polymeric microspheres [1] and soft lithography [2]. These approaches have been used to present to cells surfaces with adhesion-promoting proteins whose spatial distribution is controlled at length scales comparable to cell sizes. We are developing analogous methods based on electron-beam lithography to control cell adhesion behavior. Thusfar we have developed protocols for the e-beam patterning of biocompatible polymers and studied the adsorption of fibronectin on these.

Films of poly(DTE carbonate) [3] 100-200 nm thick were spin cast onto Si substrates from 0.1 wt\% solutions in THF. Poly(DTE carbonate) is a poly(psuedo amino acid) with attractive biophysical properties for tissue engineering applications [4] which is currently in the final stages of FDA approval. These films were annealed under a vacuum of approximately $10^{-3}$ torr at $50{ }^{\circ} \mathrm{C}$ for 1 hour to remove residual solvent. Focused-probe electron exposure was made using a LEO 982 digital FEG SEM interfaced to an Emispec Vision acquisition and control system. Emispec scripts have been written to write exposures in arbitrary patterns with controllable pixel dwell times and interpixel spacings. We typically used $10 \mathrm{keV}$ beam with currents of $0.078 \mathrm{nA}$ for $0.1 \mathrm{msec}$ pixel dwell times. After exposure, the specimens were rinsed in THF. Electron irradiation reduces the solubility of poly(DTE carbonate) in THF, presumably because of radiation-induced crosslinking. Patterned surfaces were exposed to human plasma fibronectin (FN) at $37^{\circ} \mathrm{C}$ for 2 hours in PBS-C buffer. After rinsing, the specimens were immersed in rabbit anti-human primary antibody and then flourescein-conjugated goat antirabbit IgG secondary antibody, each for $30 \mathrm{~min}$.

Figure 1 shows an SEM image of a patterned poly(DTE carbonate) surface. After development in THF, polymer dots, approximately $400 \mathrm{~nm}$ in diameter (see inset), are left at the points of e-beam exposure indicating that the poly(DTE carbonate) behaves as a negative resist. The size of the patterned poly(DTE carbonate) features is almost certainly limited by the proximity effects well known to the semiconductor industry [5]. We have thusfar achieved feature sizes as small as 100 $\mathrm{nm}$. Figure 2 shows fluorescence optical microscopy data indicating that fibronectin preferentially localizes itself at the patterned poly(DTE carbonate) features. The regions of bright contrast indicate higher concentrations of FITC-labeled antibody marking FN. We have backscattered SEM data (not shown) using $12 \mathrm{~nm}$ Au-labeled secondary antibodies which also indicatethis FN-DTE colocalization. The intensity profile in figure 2 averages 20 pixels along the direction of the line. It shows a sharp peak near its center due to the radiative effects of the incident beam. This peak has significant tails that extend several hundred nanometers away from its center. These are almost certainly due to proximity effects and polymer crosslinking from electrons backscattered from the substrate. We have not yet determined the extent of FN adsorption onto bare silicon or the extent to which poly(DTE carbonate) remains on the silicon surface after the developing THF rinse. 

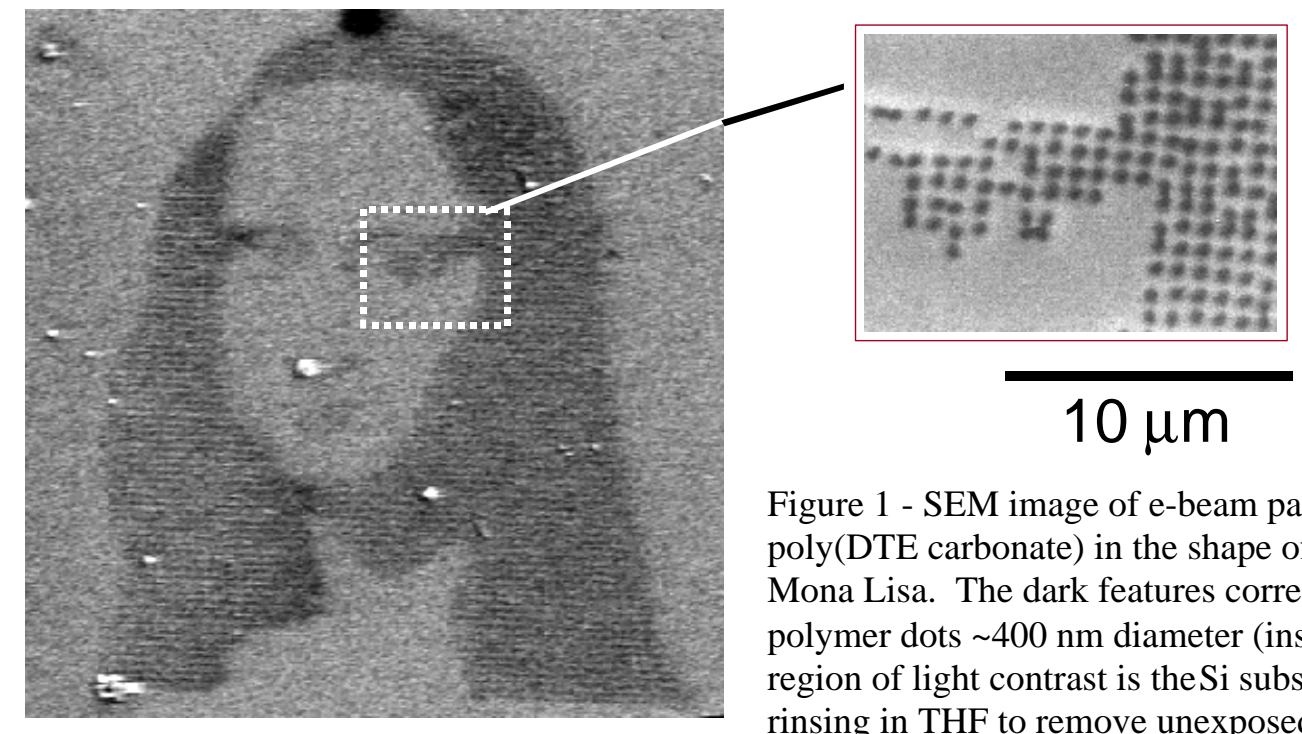

Figure 1 - SEM image of e-beam patterned poly(DTE carbonate) in the shape of the Mona Lisa. The dark features correspond to polymer dots $\sim 400 \mathrm{~nm}$ diameter (inset). The region of light contrast is the $\mathrm{Si}$ substrate after rinsing in THF to remove unexposed polymer.

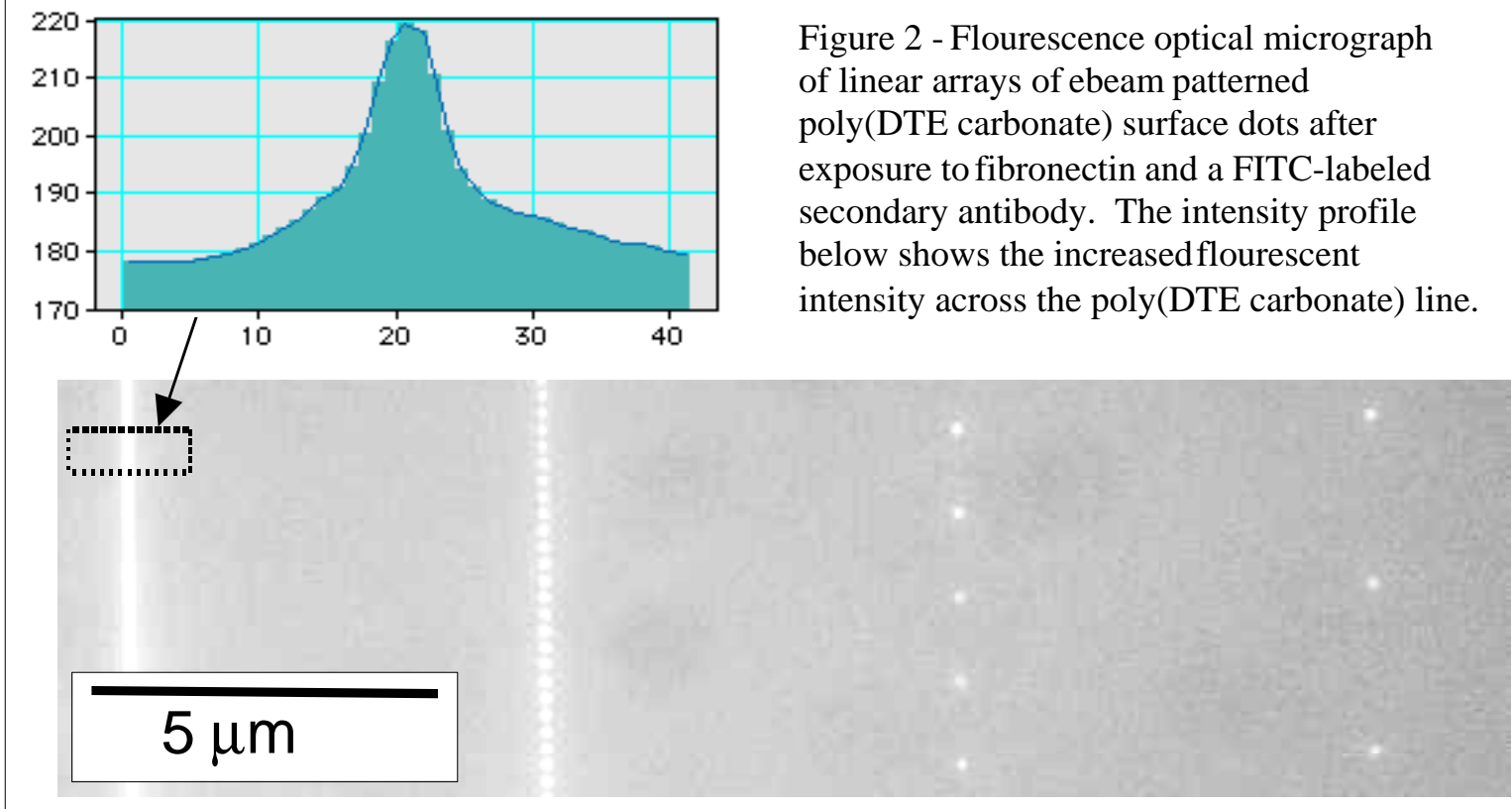

1. Folkman, J. and Moscona, A., Nature 273, 345-349 (1978).

2. $\quad$ Chen, C.S., et al., Science 276, 1425-1428 (1997).

3. Yu, C. and Kohn, J., Biomaterials 20, 253-264 (1999).

4. $\quad$ Silver, F.H., et al., J. Long-Term Effects Med. Implants 1, 329-346 (1992).

5. Rai-Choudhury, P.E., Handbook of Microlithography, Micromachining, and Microfabrication: Volume I. ( SPIE, Bellingham, Washington, 1997) 776.

6. The authors would like to acknowledge support from the Microscopy Society of America in the form of an MSA Undergraduate Research Award (P. Krsko) as well as seed funding from the New Jersey Center for Biomaterials. 